\title{
Broadband optical properties of graphene by spectroscopic ellipsometry
}

Wei Li ${ }^{1,2}$, Guangjun Cheng ${ }^{2}$, Yiran Liang ${ }^{1}$, Boyuan Tian ${ }^{1}$, Xuelei Liang ${ }^{1, *}$, Lianmao Peng $^{1}$, A.R. Hight Walker ${ }^{2}$, David J. Gundlach ${ }^{2}$, Nhan V. Nguyen ${ }^{2, *}$

1. Key Laboratory for the Physics and Chemistry of Nanodevices and Department of Electronics, Peking University, Beijing, 100871, China.

2. Physical Measurement Laboratory, National Institute of Standards and Technology, Gaithersburg, MD 20899, USA.

\begin{abstract}
The optical properties of chemical vapor deposition grown graphene were measured with high accuracy by spectroscopy ellipsometry in the energy range of 0.7 $\mathrm{eV}$ to $9.0 \mathrm{eV}$, which is spectrally broader compared with those reported in literature. The refractive index (n) and extinction coefficient (k) of graphene were accurately obtained and compared with directly measured transmittance data. The absorption of graphene follows the well-known fine structure constant in the visible range, becomes lower below $1 \mathrm{eV}$, and shows a strong absorption peak around $4.8 \mathrm{eV}$. The latter was attributed to the resonant excitons near the van Hove singularity at the M point of the Brillouin zone. A higher energy absorption peak was observed at $6.4 \mathrm{eV}$ resulting from the excitonic effect of the $\sigma$-to- $\sigma^{*}$ transition at the $\Gamma$ point of the Brillouin zone. The multi-layer graphene, fabricated by repeated transfer, exhibit similar optical properties to mono-layer graphene and the $4.8 \mathrm{eV}$ absorption peak exhibits a layer-number dependent peak shift, similar to the exfoliated AB-stacked multi-layer graphene. The broadband optical properties reported in this letter are believed to enhance the understanding of the optical properties of graphene and will benefit the development of graphene-based optoelectronic devices.
\end{abstract}

*Corresponding authors. E-mail: liangxl@pku.edu.cn (Xuelei Liang);

Nhan.Nguyen@nist.gov (Nhan V. Nguyen) 


\section{Introduction}

Graphene, one atomic thick honeycomb structure of $s p^{2}$ hybridized carbon atoms, has attracted immense attention in the worldwide scientific community since its discovery in 2004 due to its exotic quantum properties [1-3]. Electrons and holes in graphene obey the two dimensional Dirac equation and display a unique linear dispersion band structure at the K-point of the Brillouin zone [2, 3]. This unique band structure results in distinctive electronic properties such as ballistic electron transport, integer and fractional quantum Hall effect, Klein tunneling, etc. [2, 3]. Furthermore, graphene displays remarkable optical properties: highly transparency in visible spectra range which is determined by the fine structure constant $\alpha$ [4], tunable infrared optical absorbance [5, 6], and photo-response up to $\mathrm{THz}$ frequency range [7]. Consequently, graphene has found new potential applications in optics and optoelectronics.

With respect to graphene device designs and performance enhancement, it is crucial to fully understand the optical properties of graphene. The absorbance and optical conductivity of graphene have been measured by transmittance and reflectance $[8,9]$. However, other important parameters, such as the dielectric function or optical constant, are difficult to deduce from the transmittance and reflectance spectra. Several groups have attempted to obtain the complex refractive index of graphene by spectroscopy ellipsometry (SE) [10-18]. It is well-known that SE is a nondestructive and sensitive characterization method for the optical properties of materials $[19,20]$. SE measures the amplitude change $(\Psi)$ and phase shift difference $(\Delta)$ between the incident and reflected light, and the refractive index (n) and extinction coefficient (k) of a material can be extracted very accurately. With $\mathrm{n}$ and $\mathrm{k}$ other important optical parameters of a material, such as complex dielectric function, absorbance, dynamic conductivity and transmittance, can be derived accordingly.

Most of the reported optical characterization of graphene from the transmittance and reflectance measurements and SE were usually limited to below $6 \mathrm{eV}$ [8-18]. 
Higher energy irradiation will affect the properties of graphene and hence the performance of the graphene-based electronic and optoelectronic devices. It is necessary to study the optical response of graphene in a much wider energy range. Moreover, high energy optical spectra measurements will improve our understanding on the high energy resonant exciton effects and electronic band structure of graphene. In addition, previous optical measurements were commonly performed on exfoliated single crystal graphene samples which are typically very small and thus require elaborate experimental efforts [8-15, 17]. The CVD grown graphene is known to have randomly oriented grains [21], thus its optical properties are expected to be different from those of exfoliated graphene due to its polycrystalline nature. The optical constants of CVD graphene have been reported [16, 18]. However, the graphene used in these reports contains cracks and holes which affects the accuracy of the results [16]. We focus on CVD graphene here because CVD is currently the best low-cost method to produce large area graphene (up to 100 meters long [22]) with high quality and the process is considered compatible with chip fabrication, which is critical for real application of graphene devices. In this letter, broadband $(0.7 \mathrm{eV}$ to $9.0 \mathrm{eV})$ optical properties of the CVD grown graphene were measured by SE, and the corresponding optical functions were obtained with high accuracy. Higher energy absorption peak at $6.4 \mathrm{eV}$ in mono-layer graphene and weak layer-number dependence absorption of repeat-transfer multi-layer CVD graphene were observed.

\section{Experimental}

\subsection{Sample preparation}

Graphene used in this study was grown on $25 \mu \mathrm{m}$ thick $\mathrm{Cu}$ foils by the CVD method $^{1}$, and the detailed synthesis process has been described elsewhere [21]. After growth, the graphene film was transferred onto a $11 \mathrm{~mm}$ thick high quality fused

${ }^{1}$ Certain commercial equipment, instruments, or materials are identified in this report in order to specify the experimental procedure adequately. Such information is not intended to imply recommendation or endorsement by the National Institute of Standards and Technology, nor is it intended to imply that the materials or equipment identified are necessarily the best available for the purpose. 
silica substrate by modified RCA clean method developed by our group [23, 24]. This transfer method has been shown to render clean and crackless large area graphene, which is critical for accurate optical function measurements. Multi-layer (bi- and tri-layer) graphene samples were sequentially prepared by repeating the same transfer process. There are two reasons for using fused silica as substrate, instead of the

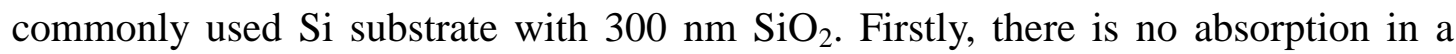
wide spectral range of the fused silica substrate [25], which simplifies the SE data analysis and allows us to extract highly accurate optical functions. Secondly, the fused silica substrate was found to enhance the measurement sensitivity of extremely thin samples such as graphene by SE simulation ${ }^{2}$.

\subsection{Characterization}

Raman spectroscopy is used to characterize the quality and uniformity of the transferred graphene. Raman measurements were performed with a Renishaw InVia ${ }^{3}$ micro-Raman spectrometer using a $514.5 \mathrm{~nm}$ wavelength excitation laser source. The spectra were measured in the backscattering configuration using a 50× objective and 1800 grooves $/ \mathrm{mm}$ grating. The spot size of the laser was approximately $1 \mu \mathrm{m}$ with an incident laser power of less than $2 \mathrm{~mW}$ to avoid any local heating effects.

SE measurements were performed in a chamber filled with nitrogen gas at room temperature on a vacuum ultraviolet spectroscopic ellipsometer consisting of Xenon and Deuterium lamps covering the wavelength from $140 \mathrm{~nm}$ to $1770 \mathrm{~nm}$ (Supporting Information). Photon energy ranging from $0.7 \mathrm{eV}$ to $9.0 \mathrm{eV}$ with $0.01 \mathrm{eV}$ step was scanned during the measurement. The ellipsometry data, $\Psi$ and $\Delta$, were taken at multiple angles of incidence.

\footnotetext{
${ }^{2}$ Li W, et al., Effective Measurement of the Optical Function of 2D Materials by Spectrospic Ellipsometry, in preparation.

${ }^{3}$ Certain commercial equipment, instruments, or materials are identified in this report in order to specify the experimental procedure adequately. Such information is not intended to imply recommendation or endorsement by the National Institute of Standards and Technology, nor is it intended to imply that the materials or equipment identified are necessarily the best available for the purpose.
} 


\section{Results and discussion}

A typical Raman spectrum of transferred CVD grown mono-layer graphene shown in Fig. 1 (a) exhibits a symmetric $\mathrm{G}^{\prime}$ peak with the intensity of about 3 times the $\mathrm{G}$ peak and no significant $\mathrm{D}$ peak $\left(\sim 1350 \mathrm{~cm}^{-1}\right)$, which confirms the high quality of the film [24] and the mono-layer thickness [26]. To identify the large area uniformity of the transferred graphene, Raman mapping was performed on a $25 \mu \mathrm{m} \times 25 \mu \mathrm{m}$ area (square marked in Fig. S3) with a step size of $1 \mu \mathrm{m}$. The maps of the integrated area of G and G' peak are shown in Fig. $1(b)$ and (c), respectively. The "hot" spots in the maps represent few-layer graphene regions [21]. Some spots are "hot" only for G or

$G^{\prime}$ peak but some are "hot" for both peaks. It is known that the Raman spectra of the bi-layer graphene is affected by the rotational-angle of the honeycomb lattice between layers [27, 28]. They could be similar to or different from the spectrum of mono-layer graphene depending on the rotation angle. The intensity variation of the "hot" spots in the Raman maps of Fig. $1(b)$ and (c) can be explained by rotational-angle dependent Raman spectra of few-layer graphene [28, 29] ( Fig. S4 in Supporting Information). By counting the pixels from the mono-layer in the mapped area, mono-layer graphene area is estimated to cover over $97.5 \%$ of the mapped area.

Typical Raman spectra of our bi-layer and tri-layer graphene are shown in Fig. $I(d)$ and (e), respectively. Four spectra from different locations were presented for each sample. These spectra are either similar (black line) to or different from (magenta, blue and red lines) that of mono-layer graphene shown in Fig. 1(a). The G' peak positions of these spectra exhibit the signature blue shift as compared with that of mono-layer graphene [27] (Fig. S5 in supporting Information). These features are reasonable because our bi-and tri-layer graphene are fabricated by repeated transfer of polycrystalline mono-layer graphene. Therefore, the lattice orientation between these layers at each location is random. 

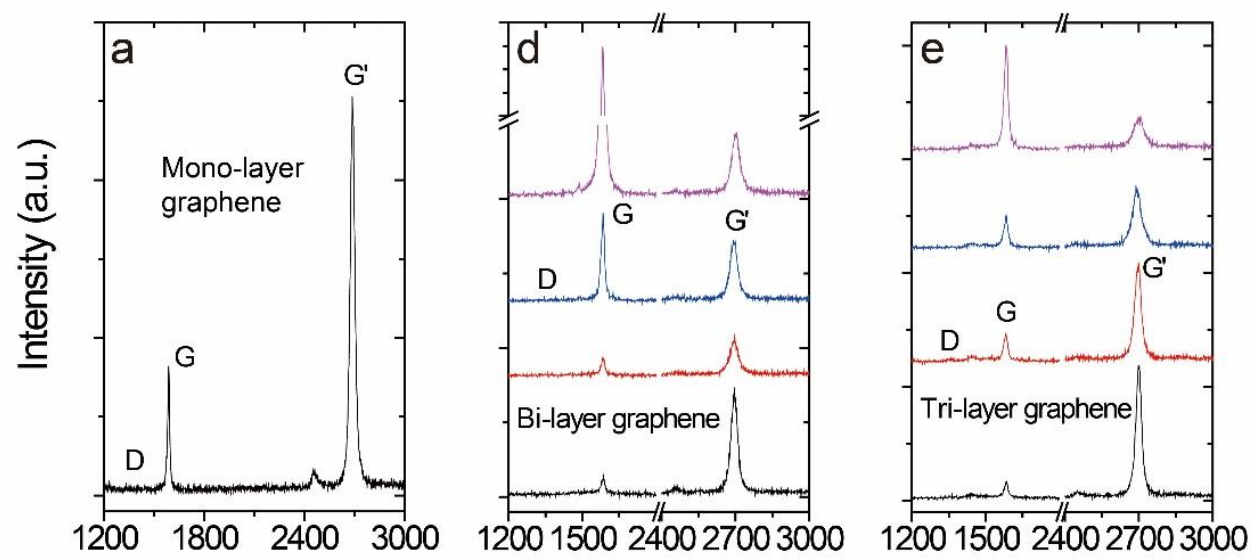

Raman shift $\left(\mathrm{cm}^{-1}\right)$

Raman shift $\left(\mathrm{cm}^{-1}\right)$

Raman shift $\left(\mathrm{cm}^{-1}\right)$
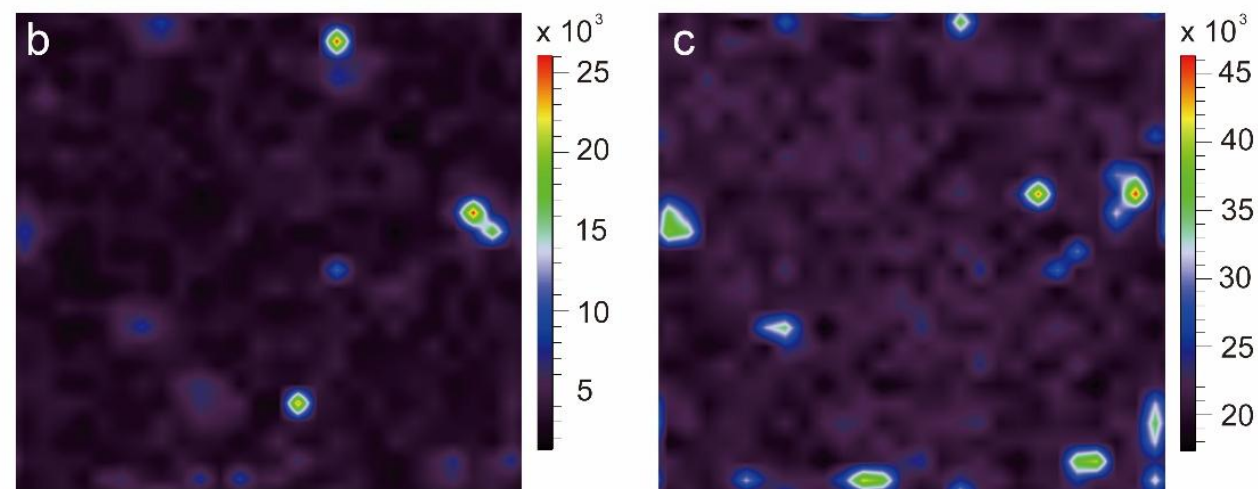

Figure 1. Raman characterization of the transferred CVD grown graphene on fused silica substrate. (a) Typical Raman spectrum of mono-layer graphene. (b) and (c) Raman maps of the integrated area of $G$ and $G^{\prime}$ peak of mono-layer graphene, respectively (taken in the white square area in Fig. S3 of the Supporting Infomation). (d) and (e) Raman spectra of bi-layer and tri-layer graphene, which were prepared by repeated transfer process. Four typical spectra from different locations are shown for each sample.
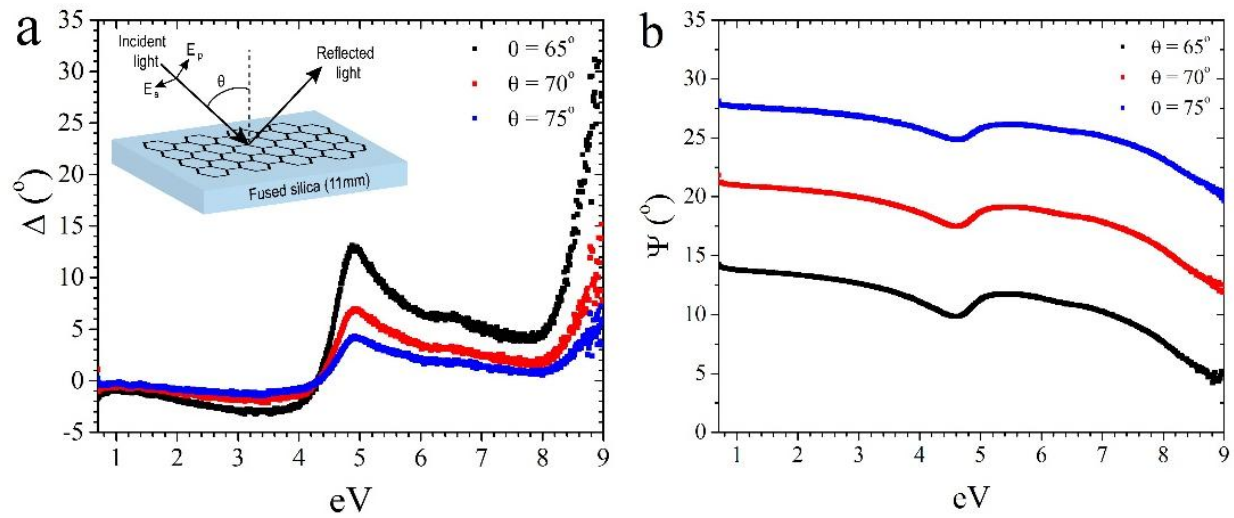

Figure 2. (a) and (b) the measured multiple angle ellipsometric parameters $\Delta$ and $\Psi$ 
of mono-layer graphene. Inset in (a): the SE measurements scheme.

Shown in Fig.2 (a) and (b) are the measured $\Delta$ and $\Psi$, respectively, for mono-layer graphene at incident angles of $65^{\circ}, 70^{\circ}$, and $75^{\circ}$. Since the fused silica was employed as the substrate, the signal-to-noise ratio of the measured $\Delta$ and $\Psi$ is relatively high. With high precision measured $\Delta$ and $\Psi$, the spectra of (n, k) are extracted (Fig. 3(a)) by least-squares fitting using the standard ellipsometric equations for a three-phase (substrate/film/ambient) model (Supporting Information). The known thickness of graphene, $3.35 \AA$, which is also the interlayer spacing of graphite, was fixed during the $(\mathrm{n}, \mathrm{k})$ extraction [15]. From $\mathrm{n}$ and $\mathrm{k}$, the corresponding absorbance was calculated [30] and displayed in Fig. 3(a). There is a peak in both $\mathrm{n}$ and $\mathrm{k}$ at the energy around $1 \mathrm{eV}$, and the calculated absorption at this energy coincides with the famous universal absorbance $\pi \alpha$, which is due to the interband transition of the massless Dirac fermions $[4,8]$. At lower energies $(<1 \mathrm{eV})$, both $\mathrm{n}$ and $\mathrm{k}$ decrease with decreasing energy resulting in lower absorption. Such absorption lowering has been investigated and attributed to the effects of the doping-induced Pauli blocking of interband transitions, i.e., doping in the graphene film causing a shift of the Fermi energy $\mathrm{E}_{\mathrm{F}}$ away from the Dirac point, and thus blocking the interband transitions for photon energies below $2\left|\mathrm{E}_{\mathrm{F}}\right|[8]$. At energies above $1 \mathrm{eV}$, with the increasing photon energy, the extinction coefficient $k$ first decreases and then increases and peaks at 4.8 $\mathrm{eV}$, while $\mathrm{n}$ shows a decrease around this energy. This leads to a prominent asymmetric peak in the absorption at $4.6 \mathrm{eV}$, which is a well-known feature resulting from the resonance excitons near the saddle-point singularity of $M$ point in the Brillouin zone [9, 15, 31, 32].

Fig. 3(b) and (c) show the comparison of (n, k) spectra from this study with published SE measurement results. The overall trends of spectral response of both $n$ and $\mathrm{k}$ are similar in the energy range from $1.4 \mathrm{eV}$ to $5 \mathrm{eV}$. The discrepancy between our data and the reported spectra is likely due to the differences in sample preparation (e.g. exfoliated graphene on $\mathrm{SiO}_{2}[10,11,15]$, or amorphous quartz [15], or cracked 
CVD graphene on glass [16]) and/or the data processing methods (e.g. B-spline parameterization [10] or dispersion modeling [15, 17]). Recently, Cheon et al. [33] determined the $(\mathrm{n}, \mathrm{k})$ of mono-layer graphene which is $(2.58 \pm 0.38,1.3 \pm 0.25)$ at 634 $\mathrm{nm}$ by combining the surface plasmon resonance angle detection and the attenuated total reflection intensity measurements. At the same wavelength, our results is $(2.91$, 1.51), which is consistent with Cheon et al.'s results within their measurement accuracy as shown in Fig. 3(a). Furthermore, the published calculation using density function theory (DFT) yielded the values of $(2.96,1.49)$ at $634 \mathrm{~nm}$ [34], which are much closer to $\mathrm{n}$ and $\mathrm{k}$ from our report than those of Cheon et al. Even though in principle, (n, k) can be determined with high precision in Cheon et al.'s experiment, their data are limited to only a single wavelength. Therefore, our results provide high accuracy (n, k) spectra over a broad band range, which are more favorable and advantageous for graphene optoelectronic devices design.
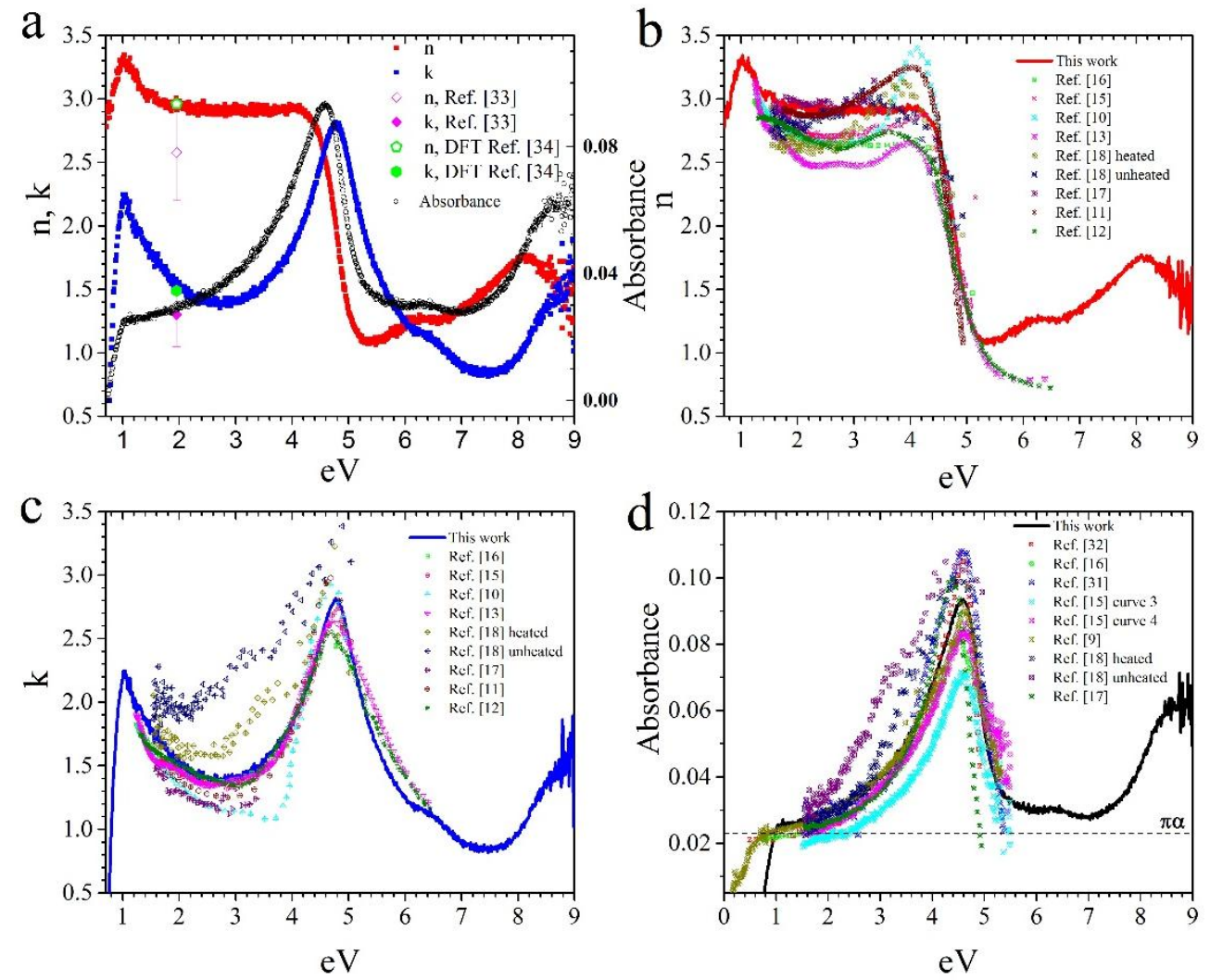

Figure 3 Spectroscopic ellipsometry data of CVD grown mono-layer graphene on fused silica substrate. (a) The extracted $(n, k)$ and absorbance of mono-layer CVD grown graphene, as well as the experimental [33] and calculated [34] data at $634 \mathrm{~nm}$ wavelength. (b-d) The 
comparison of our extracted $n, k$ and absorbance with those reported by other groups (see text).

We also compared the absorbance previously reported with our results in Fig. 3(d). All of the absorbance spectra show a similar trend. However, the position, intensity and broadening of the exciton-related asymmetric absorption peak at $4.6 \mathrm{eV}$ show some variation. The variation may be caused by the different dielectric conditions and screening effects on the exciton for different substrates. Moreover, structural imperfections (grain boundaries, defects, residues from transfer, etc.) may exist more in CVD grown graphene than in exfoliated graphene used by other groups. For energies below $1 \mathrm{eV}$, the absorbance decreases with decreasing energy. However, our results begin to decrease at a higher energy as compared with those of Mak et al. [9]. As discussed in the above, this absorption lowering is due to the doping effect and higher doping level results in higher threshold energy of the absorbance decreasing. It is likely that our CVD graphene was unintentionally doped while being transferred onto the fused silica substrate $[16,24,35]$.

The optical properties of graphene reported in literature are mainly limited to the photon energy below $6 \mathrm{eV}$, while at higher energy range the reported values of $\mathrm{n}$ and $\mathrm{k}$ which can be considered reliable are rather limited to only a few spectral points [8-18]. It is considered more desirable and important for understanding of the electronic structures of graphene as well as graphene optoelectronic device designs and application to have access to a broader and more detailed spectral response. In our study, we extended the measured energy range up to $9.0 \mathrm{eV}$ and thus more spectral features such as the absorption peak at $6.4 \mathrm{eV}$ can be clearly observed. Currently, only Santoso et al. experimentally reported an absorption peak at $6.3 \mathrm{eV}$ in epitaxial graphene on $\mathrm{SiC}$ [36]. Trevisanutto et al. have predicted a high energy absorption peak at $8.3 \mathrm{eV}$ due to the resonant excitons between the $\sigma$-to- $\sigma^{*}$ transition at the $\Gamma$ point of the Brillouin zone by ab initio many-body calculations [37]. However, the interaction between graphene and the substrate was neglected in their calculations. 
When taking into account of this interaction using the density function theory (DFT), Santoso et al. have found that the $\sigma$-to- $\sigma^{*}$ resonant excitons redshifted from $8.3 \mathrm{eV}$ to $6.3 \mathrm{eV}$. The interaction was modeled as the charge transfer from the $\mathrm{SiC}$ substrate to graphene and the effects of the lattice mismatch strain [36]. Since our graphene sample was transferred onto the substrate instead of a direct growth, the graphene/substrate interaction can be considered negligible and therefore the redshift may not be caused by the interaction as suggested in Santoso et al.'s interpretation. This suggests that further theoretical work is needed to elucidate the new optical and electronic structure of graphene at higher energy. At the energies above $7 \mathrm{eV}$, the absorption increases gradually and another absorption peak seems appearing at $8.5 \mathrm{eV}$. Since the data became more noisy and the band gap absorption of the fused silica substrate also began at this energy range [25]. It cannot be unambiguously claim an absorption peak exists in this energy range. Nonetheless, it can be concluded that the increase of absorption below $8.5 \mathrm{eV}$ originates from graphene excluding any optical artifacts which may arise from the fused silica substrate.

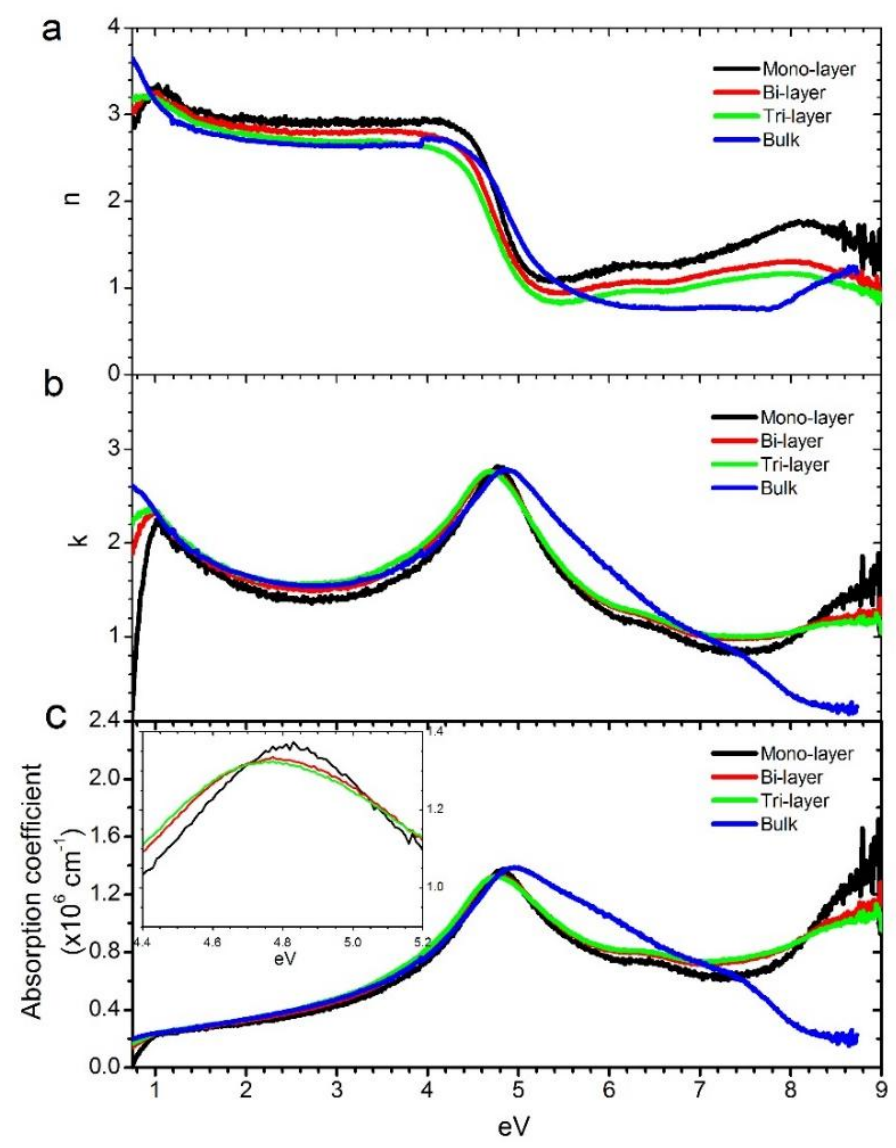


Figure 4. The extracted n (a), k (b), and absorption coefficient (c) for mono-, bi- and tri-layer CVD grown graphene. The inset figure in (c) is a zoom-in portion of the peak at about $4.8 \mathrm{eV}$.

In recent studies, the optical properties of exfoliated multi-layer graphene were shown to contain a few special optical features which are found to be different for both mono-layer graphene and graphite. With the significantly improved CVD method, multi-layer CVD grown graphene can also be fabricated by repeating the transfer process and it is necessary to fully understand the optical properties of such multi-layer graphene. Such studies are believed not to have been carried out. Here, we present our findings on bi- and tri-layer graphene fabricated by repeated transfer. Fig. 4 displays $\mathrm{n}$ and $\mathrm{k}$ extracted from ellipsometry measurements on mono-layer (3.35 $)$ ), bi-layer $(6.7 \AA)$ and tri-layer (10.05 $\AA$ ) graphene along with bulk graphite used as a reference. The (n, k) and absorption coefficient spectra of the mono-, bi-, tri-layer graphene show a very similar trend but quite different characteristics from those of the bulk graphite. At low energies below $1 \mathrm{eV}$, both $\mathrm{n}$ and $\mathrm{k}$ (blue curve) of the graphite increase as the energy decreases whereas those of graphene decrease. The absorption peak around $4.8 \mathrm{eV}$ is more symmetric in bulk graphite than in graphene due to higher screening effect in bulk. With our measurements, the absorption peak at $6.4 \mathrm{eV}$ in mono-layer graphene can be clearly observed and it shifts to $\sim 7.5 \mathrm{eV}$ in graphite, indicating a large binding energy of the resonant excitons of the $\sigma$-to- $\sigma *$ transition in graphene. In contrast to the mono-layer and multi-layer graphene, the $\mathrm{k}$ value of the bulk monotonically decreases from $7.5 \mathrm{eV}$ to $8.5 \mathrm{eV}$. It is known that the optical properties of graphene are layer-dependent $[9,32,36]$, and the absorption in exfoliated multi-layer graphene displays oscillations in the low energy range $(<1 \mathrm{eV})$ due to the interlayer interaction $[9,32]$. However, such oscillations were not observed in our bi- and tri-layered graphene. This can be explained by the fact that our samples were fabricated by repeated transfer and the interlayer interaction is much weaker than that of graphite or exfoliated multi-layer graphene. Furthermore, in comparison with exfoliated AB-stacked multi-layer graphene, our multi-layer graphene sample is a mixture of different lattice orientation between the layers. In fact, our bi- and 
tri-layer samples are simply stacking of mono-layer graphene and physically its properties are expected to be almost the same as mono-layer graphene. Interestingly, the exciton-related absorption peak at $\sim 4.6 \mathrm{eV}$ for bi-layer and tri-layer samples slightly redshift about $40 \mathrm{meV}$ and $60 \mathrm{meV}$ relative to that of the mono-layer graphene. This layer number-dependent redshift has been observed in exfoliated multi-layer graphene samples and explained as the effective cancellation of the comparable repulsive electron-electron and attractive electron-hole interactions [9]. Our results clearly show that such peak shift occurs even in our non-AB stacking multi-layer graphene samples.

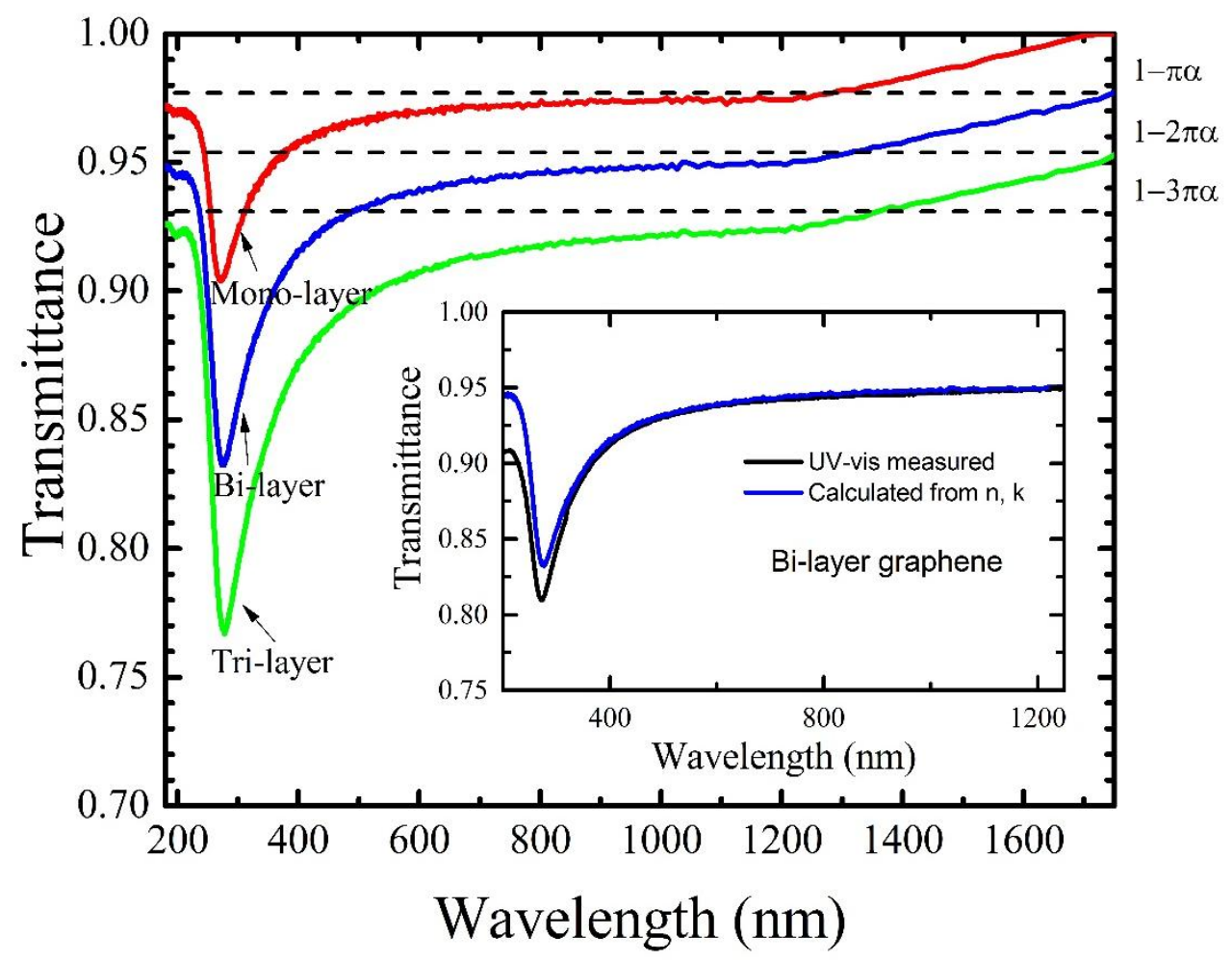

Figure 5. (a) The calculated transmittance of mono-, bi- and tri-layer CVD grown graphene. The inset shows the comparison of measured transmittance and calculated transmittance for bi-layer CVD grown graphene.

With $(n, k)$ of different layers of graphene obtained, the transmittance spectra can be calculated and the results are shown in Fig. 5. The transmittance of mono-, bi- and tri-layer graphene are $97.5 \%, 94.9 \%$ and $92.4 \%$, respectively, at and in the vicinity of $1000 \mathrm{~nm}$ wavelength show minor deviations from that of the suspended exfoliated 
graphene [4]. This deviation is mainly due to chemical residues unintentionally introduced during the transfer process of the CVD-grown graphene [18, 24, 35]. From $600 \mathrm{~nm}$ to $1200 \mathrm{~nm}$, the transmittance remains almost a constant with the magnitude of $1-\mathrm{N} \pi \alpha$ where $\mathrm{N}$ is the number of graphene layers. Outside this spectral range, the transmittance deviates from the constant. To confirm our measurement consistency, we also performed the transmittance measurement as commonly done and reported in literature [38]. A typical comparison of bi-layer graphene is shown in the inset of Fig. 5. The two spectra are very consistent with each other, in particular at wavelengths longer than $300 \mathrm{~nm}$. Since the UV-vis transmittance is run in the air and the UV light will be partly absorbed in air, what's more, the reflectance ellipsometry measurement is more sensitive to the surface than the transmittance, and the UV light has a shorter penetration depth, the deviation between the two measurements is expected at wavelengths below $300 \mathrm{~nm}$.

\section{Summary}

In summary, the broad band $(0.7 \mathrm{eV}$ to $9.0 \mathrm{eV})$ optical properties of CVD grown graphene are measured by spectroscopic ellipsometry. The absorbance decreases with energies below $1 \mathrm{eV}$ and becomes nearly a constant in the visible range. The exciton-related absorption peak at $4.8 \mathrm{eV}$ is observed, consistent with previous reports. Moreover, an absorption peak at about $6.4 \mathrm{eV}$ was also observed and associated with the resonant excitons of the $\sigma$-to- $\sigma^{*}$ transition at the $\Gamma$ point in the Brillouin zone. Interestingly and significantly, in the multi-layered graphene fabricated using a repeated transfer method, the $4.8 \mathrm{eV}$ peak exhibits a layer-number dependence which was previously observed in the exfoliated AB-stacked multi-layer graphene. The optical properties reported in this letter can be considered a valuable contribution to further the understanding of graphene as well as to assist the development of graphene-based optical and optoelectronic devices.

\section{Acknowledgements}

This work was supported by the Ministry of Science and Technology of China 
(Grant No. 2011CB921904), the Ministry of education of China (Grant No. 113003A), Natural Science Foundation of China (Grant No. 61321001) and Beijing Municipal Science \& Technology Commission (Grant No. Z141100003814006). Wei Li is partly supported by National Institute of Standards and Technology. The authors are grateful to the discussion with Alex Boosalis of University of Nebraska-Lincoln. 


\section{Reference}

[1] Novoselov KS, Geim AK, Morozov SV, Jiang D, Zhang Y, Dubonos SV, et al. Electric Field Effect in Atomically Thin Carbon Films. Science. 2004;306(5696):666-9.

[2] Geim AK, Novoselov KS. The rise of graphene. Nature materials. 2007;6(3):183-91.

[3] Castro Neto AH, Guinea F, Peres NMR, Novoselov KS, Geim AK. The electronic properties of graphene. Rev Mod Phys. 2009;81(1):109-62.

[4] Nair RR, Blake P, Grigorenko AN, Novoselov KS, Booth TJ, Stauber T, et al. Fine Structure Constant Defines Visual Transparency of Graphene. Science. 2008;320(5881):1.

[5] Chen CF, Park CH, Boudouris BW, Horng J, Geng B, Girit C, et al. Controlling inelastic light scattering quantum pathways in graphene. Nature. 2011;471(7340):617-20.

[6] Li ZQ, Henriksen EA, Jiang Z, Hao Z, Martin MC, Kim P, et al. Dirac charge dynamics in graphene by infrared spectroscopy. Nature Physics. 2008;4(7):532-5.

[7] Xia F, Thomas M, Lin Y-m, Valdes-Garcia A, Avouris P. Ultrafast graphene photodetector. Nature Nanotechnology. 2009;4(12):839-43.

[8] Mak KF, Sfeir MY, Wu Y, Lui CH, Misewich JA, Heinz TF. Measurement of the Optical Conductivity of Graphene. Physical Review Letters. 2008;101(19):196405.

[9] Mak KF, Shan J, Heinz TF. Seeing Many-Body Effects in Single- and Few-Layer Graphene: Observation of Two-Dimensional Saddle-Point Excitons. Physical Review Letters. 2011;106(4):046401.

[10] Weber JW, Calado VE, van de Sanden MCM. Optical constants of graphene measured by spectroscopic ellipsometry. Applied Physics Letters. 2010;97(9):091904.

[11] Matković A, Ralević U, Isić G, Jakovljević MM, Vasić B, Milošević I, et al. Spectroscopic ellipsometry and the Fano resonance modeling of graphene optical parameters. Physica Scripta. 2012;2012(T149):014069.

[12] Wang W, Balooch M, Claypool C, Zawaideh M, Farnaam K. Combined reflectometry-ellipsometry technique to measure graphite down to monolayer thickness. Solid State Technol. 2009;52(6):18-21.

[13] Gray A, Balooch M, Allegret S, De Gendt S, Wang W-E. Optical detection and characterization of graphene by broadband spectrophotometry. Journal of Applied Physics. 2008;104(5):053109.

[14] Isić G, Jakovljevic M, Filipovic M, Jovanovic D, Vasic B, Lazovic S, et al. Spectroscopic ellipsometry of few-layer graphene. Journal of Nanophotonics. 2011;5(1):051809.

[15] Kravets VG, Grigorenko AN, Nair RR, Blake P, Anissimova S, Novoselov KS, et al. Spectroscopic ellipsometry of graphene and an exciton-shifted van Hove peak in absorption. Physical Review B. 2010;81(15):155413.

[16] Nelson FJ, Kamineni VK, Zhang T, Comfort ES, Lee JU, Diebold AC. Optical properties of large-area polycrystalline chemical vapor deposited graphene by spectroscopic ellipsometry. Applied Physics Letters. 2010;97(25):253110-112.

[17] Matković A, Beltaos A, Milićević M, Ralević U, Vasić B, Jovanović D, et al. Spectroscopic imaging ellipsometry and Fano resonance modeling of graphene. Journal of Applied Physics. 2012;112(12):123523.

[18] Matković A, Ralević U, Chhikara M, Jakovljević MM, Jovanović D, Bratina G, et al. Influence of transfer residue on the optical properties of chemical vapor deposited graphene investigated through spectroscopic ellipsometry. Journal of Applied Physics. 2013;114(9):093505.

[19] Aspnes DE. Optical properties of solids. 1 ed. Balkanski M, editor. New York: North-Holland Publishing Company; 1982. 
[20] Azzam RMA, Bashara NM. Ellipsometry and Polarized Light. Amsterdam: Elsevier Science B. V.; 1977.

[21] Li X, Cai W, An J, Kim S, Nah J, Yang D, et al. Large-Area Synthesis of HIgh-Quality and Uniform Graphene Films on Copper Foils. Science. 2009;324(5932):1312-14.

[22] Kobayashi T, Bando M, Kimura N, Shimizu K, Kadono K, Umezu N, et al. Production of a 100-m-long high-quality graphene transparent conductive film by roll-to-roll chemical vapor deposition and transfer process. Applied Physics Letters. 2013;102(2):023112.

[23] Liang X, Sperling BA, Calizo I, Cheng G, Hacker CA, Obeng Y, et al. Toward Clean and Crackless Transfer of Graphene. ACS Nano. 2011;5(11):9144-53.

[24] Liang X, Li W, Cheng G, Calizo I, Hacker CA, Hight Walker AR, et al. The effects of the transfer process on the quality of CVD-grown graphene Chin Sci Bull. 2014;59(33):3322-8.

[25] Li W, Birdwell AG, Amani M, Burke RA, Ling X, Lee $\mathrm{Y}-\mathrm{H}$, et al. Broadband optical properties of large-area monolayer CVD molybdenum disulfide. Physical Review B. 2014;90(19):195434.

[26] Ferrari AC, Meyer JC, Scardaci V, Casiraghi C, Lazzeri M, Mauri F, et al. Raman Spectrum of Graphene and Graphene Layers. Physical Review Letters. 2006;97(18):187401.

[27] Kim K, Coh S, Tan LZ, Regan W, Yuk JM, Chatterjee E, et al. Raman Spectroscopy Study of Rotated Double-Layer Graphene: Misorientation-Angle Dependence of Electronic Structure. Physical Review Letters. 2012;108(24):246103.

[28] Havener RW, Zhuang H, Brown L, Hennig RG, Park J. Angle-resolved Raman imaging of interlayer rotations and interactions in twisted bilayer graphene. Nano Lett. 2012;12(6):3162-7.

[29] Fang W, Hsu AL, Caudillo R, Song Y, Birdwell AG, Zakar E, et al. Rapid identification of stacking orientation in isotopically labeled chemical-vapor grown bilayer graphene by Raman spectroscopy. Nano Lett. 2013;13(4):1541-8.

[30] Heavens OS. Optical Properties of Thin Solid Films. North Chelmsford: Dover Publications; 2nd edition 2011.

[31] Chae DH, Utikal T, Weisenburger S, Giessen H, Klitzing KV, Lippitz M, et al. Excitonic fano resonance in free-standing graphene. Nano Lett. 2011;11(3):1379-82.

[32] Yang L, Deslippe J, Park C-H, Cohen ML, Louie SG. Excitonic Effects on the Optical Response of Graphene and Bilayer Graphene. Physical Review Letters. 2009;103(18):186802.

[33] Cheon S, Kihm KD, Kim Hg, Lim G, Park JS, Lee JS. How to reliably determine the complex refractive index (RI) of graphene by using two independent measurement constraints. Scientific reports. 2014;4:06364.

[34] Klintenberg M, Lebegue S, Ortiz C, Sanyal B, Fransson J, Eriksson O. Evolving properties of two-dimensional materials: from graphene to graphite. J Phys: Condens Matter. 2009;21(33):335502.

[35] Ishigami M, Chen JH, Cullen WG, Fuhrer MS, Williams ED. Atomic Structure of Graphene on SiO2. Nano Lett. 2007;7(6):1643-8.

[36] Santoso I, Gogoi PK, Su HB, Huang H, Lu Y, Qi D, et al. Observation of room-temperature high-energy resonant excitonic effects in graphene. Physical Review B. 2011;84(8):081403.

[37] Trevisanutto PE, Holzmann $M$, Cote $M$, Olevano $V$. Ab initio high-energy excitonic effects in graphite and graphene. Physical Review B. 2010;81(12):121405.

[38] Zhu S-e, yuan S, Janssen GCAM. Optical transmittance of multilayer graphene. EPL (Europhys Lett). 2014;108(1):17007. 